\title{
Bi-directional switching based on semiconductor laser/amplifier with shallow-etched bending ridge waveguide
}

\author{
Ching-Fuh Lin ${ }^{\text {a) }}$ and Bor-Lin Lee \\ Institute of Electro-Optical Engineering and Department of Electrical Engineering, National Taiwan \\ University, Taipei, Taiwan, Republic of China
}

(Received 4 March 1997; accepted for publication 4 August 1997)

\begin{abstract}
A new type of optical switching device is developed. For a semiconductor laser amplifier with a shallow-etched bending ridge waveguide, the laser beam could propagate along the bending direction or the straight direction. Switching between the two directions is characterized. With the device fabricated on the substrate with two quantum wells of different widths, switching characteristics are found to significantly depend on the spectral separation of the two lasing modes.

(C) 1997 American Institute of Physics. [S0003-6951(97)02140-2]
\end{abstract}

Two-mode optical switching has potential applications in optical communications and optical signal processing. ${ }^{1-3}$ The two oscillating modes may be two wavelengths, ${ }^{2} \mathrm{TE}$ and TM polarization states, ${ }^{4-5}$ or two spatially distinct, but cross coupled laser diodes. ${ }^{6-8}$ Among them, the spatially distinct laser output is preferred because of its compatibility with intensity modulated optical signals. Although efficient switching has been achieved in spatially distinct cross coupled laser diodes, ${ }^{7,8}$ complicated structures and processing techniques are required therein. In this work, we report a new type of two spatially distinct mode switching. We use a weakly index-guided bending waveguide to provide a simple and efficient method for switching between the bending direction and the straight-propagation direction. Switching between the two directions is characterized. With the devices fabricated on the substrate with two quantum wells of different widths, switching characteristics significantly depend on the spectral separation of the two lasing modes.

The devices were fabricated on a graded index separated confinement heterostructure (GRINSCH) substrate with dual GaAs quantum wells of different widths separated by a 150 $\AA \mathrm{Al}_{0.18} \mathrm{Ga}_{0.82} \mathrm{As}$ barrier layer. Figure 1 shows the layer structure of the substrate. A schematic of the device is shown in Fig. 2(a). It has a 5- $\mu \mathrm{m}$-wide ridge waveguide that consists of a $200 \mu \mathrm{m}$ straight section and a $\sim 500 \mu \mathrm{m}$ bending section. The bending section is formed by 35 segments that connect one after another. Each segment is oriented at an increasing angle with respect to the direction of the straight section. The segment nearest to the output facet is oriented at an angle of $7^{\circ}$ from the normal of the cleaved facet. Chemical etching was used to create the ridge structure, but was stopped at about $0.7 \mu \mathrm{m}$ above the active layer. $\mathrm{A} \mathrm{SiO}_{2}$ insulation layer was deposited after the etching. Then, this layer was etched away on top of the ridge waveguide for $p$-contact metallization. The fabrication was completed by $n$-contact metallization and the devices were then cleaved apart.

The shallow-etched depth leads to a small difference of the refractive indices between the ridge and its neighboring regions, so the guiding effect of the ridge waveguide is weak. The weak guiding effect then causes the light to possibly propagate along two paths. The reason is explained as

${ }^{a)}$ Electronic mail: cflin@cc.ee.ntu.edu.tw follows. Due to the weak guiding effect, the light will not be well guided along the bending section when it propagates from the straight section to the bending section. Some portion of the light continues to propagate along the straight path, as indicated by the dashed lines in Fig. 2(a), while the other portion is still guided along the bending ridge waveguide. Two near-field beam spots could then be observed at the output facet of the bending ridge waveguide. One corresponds to the bending mode and the other corresponds to the straight-propagation mode.

Figures 2(b) and 2(c) show the near-field output beam spots of the device measured by a charge coupled device (CCD) camera for the injection current below and above 103 $\mathrm{mA}$, respectively. This observation shows the switching from the bending mode to the straight-propagation mode as the injection current increases. These beam spots were not well focused due to the limitation of our optical setup. The left

\begin{tabular}{|c|c|c|c|}
\hline 1 & GaAs & $\mathrm{p}=5^{*} 10^{18} \mathrm{~cm}^{-3}$ & $0.25 \mu \mathrm{m}$ \\
\hline 2 & $\begin{array}{c}\text { Graded } \mathrm{Al}_{\mathrm{x}} \mathrm{Ga}_{1-\mathrm{x}} \mathrm{As} \\
\mathrm{x}=0.5-0.2\end{array}$ & $p=10^{18} \mathrm{~cm}^{-3}$ & $800 \AA$ \\
\hline 3 & $\mathrm{Al}_{0.5} \mathrm{Ga}_{0.5} \mathrm{As}$ & $\mathrm{p}=9 * 10^{17} \mathrm{~cm}^{-3}$ & $1.5 \mu \mathrm{m}$ \\
\hline 4 & $\begin{array}{c}\text { Graded } \mathrm{Al}_{\mathrm{x}} \mathrm{Ga}_{1-\mathrm{x}} \mathrm{As} \\
\mathrm{x}=0.18-0.5\end{array}$ & undoped & $2000 \AA$ \\
\hline 5 & GaAs & undoped & $75 \AA$ \\
\hline 6 & $\mathrm{Al}_{0.18} \mathrm{Ga}_{0.82} \mathrm{As}$ & undoped & $150 \AA$ \\
\hline 7 & GaAs & undoped & $40 \AA$ \\
\hline 8 & $\begin{array}{c}\text { Graded } \mathrm{Al}_{\mathrm{x}} \mathrm{Ga}_{1-\mathrm{x}} \mathrm{As} \\
\mathrm{x}=0.5-0.18\end{array}$ & undoped & $2000 \AA$ \\
\hline 9 & $\mathrm{Al}_{0.5} \mathrm{Ga}_{0.5} \mathrm{As}$ & $\mathrm{n}=7^{*} 10^{17} \mathrm{~cm}^{-3}$ & $1.5 \mu \mathrm{m}$ \\
\hline 10 & $\begin{array}{c}\text { Graded } \mathrm{Al}_{\mathrm{x}} \mathrm{Ga}_{1-\mathrm{x}} \mathrm{As} \\
\quad \mathrm{x}=0.2-0.5\end{array}$ & $\mathrm{n}=10^{18} \mathrm{~cm}^{-3}$ & $1000 \AA$ \\
\hline 11 & GaAs & $\mathrm{n}=10^{19} \mathrm{~cm}^{-3}$ & $0.5 \mu \mathrm{m}$ \\
\hline
\end{tabular}

12 n-GaAs substrate

FIG. 1. The layer structure of the substrate with dual quantum wells of different widths. 
(a)

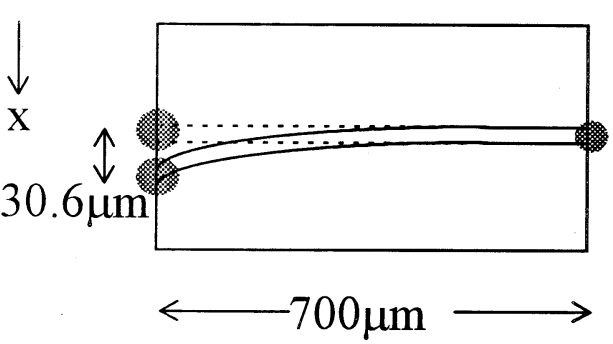

(b)

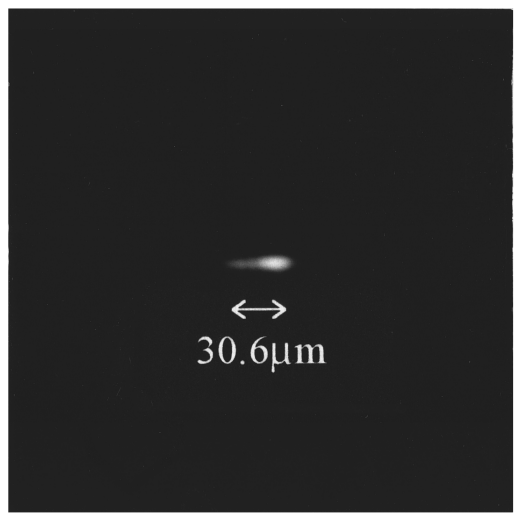

$\mathbf{X}$

(c)

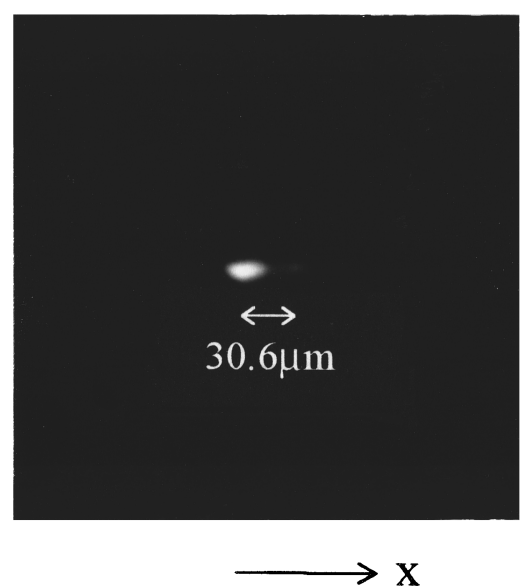

FIG. 2. (a) A schematic of the optical switching device with bending waveguide. (b) The beam spots at the output facet of the device at low injection current. (c) The same beam spots at large injection current: (left spot) straight-propagation mode; (right spot) bending mode.

and right beam spots are emitted from the straightpropagation path and the bending waveguide, respectively. The centers of the two light spots are separated for about 30 $\mu \mathrm{m}$ because the bending waveguide deviates from the straight-propagation path for $\sim 30 \mu \mathrm{m}$ at the output facet. At the low injection current, the straight-propagation mode is observed to be dimmer than the bending mode for two reasons. First, this light experiences more loss because there is no direct contact between the metal and the highly doped $p$-type GaAs layer along the straight-propagation path indicated by the dashed lines in Fig. 2(a), and thus no current is directly injected into this region. Second, because the light is mainly polarized along the junction plane, it is less transmitted at the normal incidence. As the injection current in-

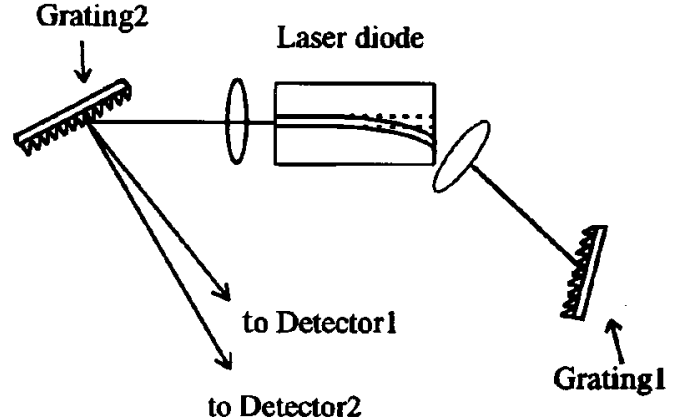

FIG. 3. The experimental setup of the external-cavity semiconductor laser for observing the switching between the bending mode and the straightpropagation mode.

creases to $103 \mathrm{~mA}$, the straight-propagation mode starts to lase at around $848 \mathrm{~nm}$, and this mode becomes much more intense than the bending mode. It appears to be impossible to have the light lasing along the path indicated by the dashed lines in Fig. 2(a) because this path is not directly pumped. However, the large radius of curvature of the bending ridge waveguide makes it stay closer to the straight-propagation path for quite a long distance. Then the large current spreading in the shallow-etched ridge waveguide ${ }^{9}$ causes the straight-propagation path that is close to the pumped bending waveguide to be partially pumped. Therefore, resonance along this path between the two cleaved facets could occur.

Switching from the straight-propagation mode to the bending mode is also observed. We used an external grating to form a laser cavity for the bending waveguide. The experimental setup is shown in Fig. 3. Because the waveguide is oriented at an oblique angle to the cleaved facet, very little retro-reflection is experienced at this output facet. ${ }^{10}$ Therefore, the lasing wavelength of the bending mode in the external-cavity configuration is widely tunable. On the other hand, the straight-propagation mode remains at $\sim 848 \mathrm{~nm}$. An additional grating is used in the output of the externalcavity configuration to separate the straight-propagation mode and the bending mode. Figure 4 shows the measured $L-I$ curve corresponding to such a switching between these two modes. The straight-propagation mode oscillates first with a threshold current of $103 \mathrm{~mA}$. When the pumping current reaches $116 \mathrm{~mA}$, the bending mode starts to lase. In this measurement, the bending mode is tuned to the wavelength of $835 \mathrm{~nm}$. Because the external grating returns more power than the cleaved facet, the bending mode could be more intense and then quenches the straight-propagation mode as a result of the gain competition between the two modes. Such a switching could be explained by the two-mode competition theory. ${ }^{11}$ For practical use of the device in switching applications, the two beam spots of the straight-propagation mode and the bending mode need to be coupled out separately. This could possibly be achieved by using fiber coupling techniques or integrating this device with other optical components.

It is discovered that the switching characteristics depend on the spectral separation of the two lasing modes. Because the device is fabricated on the substrate with dual quantum wells of different widths, the wavelength of the bending mode can be tuned over $40 \mathrm{~nm} .{ }^{12}$ Carriers in both quantum 


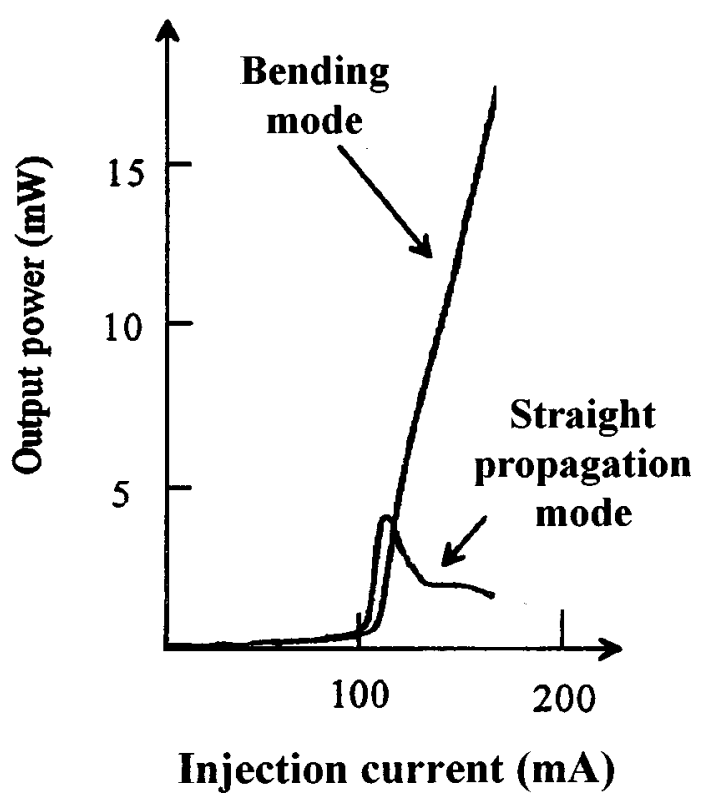

FIG. 4. $L-I$ curves of the optical switching device with the bending mode tuned to the wavelength $835 \mathrm{~nm}$.

wells could contribute to the stimulated emission. The $n=1$ transitions of the two wells separate for about $50 \mathrm{meV}$, which corresponds to $\sim 30 \mathrm{~nm}$. The lasing wavelength 848 $\mathrm{nm}$ of the straight-propagation mode indicates that it is mainly contributed from the carriers in the wide quantum well. For the bending mode with a wavelength from 840 to $855 \mathrm{~nm}$, its main contribution also comes from the carriers in the wide quantum well. In this spectral range, the bending mode has a lower threshold current than the straightpropagation mode. Because the gain in the shared gain region is always first depleted by the bending mode as long as the oscillation starts, the straight-propagation mode obtains only negligible gain and has no chance to oscillate.

For the bending mode lasing at a wavelength between 827.5 and $840 \mathrm{~nm}$, carriers in both wells could contribute to this mode. The switching characteristics for this spectral range are similar to that measured at $835 \mathrm{~nm}$ except that the bending mode has a threshold current slightly different from $116 \mathrm{~mA}$. Gain competition of the two modes is the main reason for such a switching. The physics behind the gain competition should result from the strong interaction of carriers in the same quantum well because the carriers in the wide quantum well simultaneously contribute to both modes.

For the bending mode with a wavelength smaller than $827.5 \mathrm{~nm}$, it is contributed mainly from carriers in the narrow quantum well. In this case, carriers contributing to the bending mode and the straight-propagation mode come from two uncoupled quantum wells, ${ }^{12}$ respectively. Because the interaction of carriers belonging to different wells is relatively weak, no strong gain competition between these two modes is experienced. As a result, the straight-propagation mode is not quenched although it is still influenced by the bending mode, as shown in Fig. 5. In this measurement, the bending mode lases at $822.5 \mathrm{~nm}$. For the bending mode lasing at a shorter wavelength, the straight-propagation mode is even less influenced.

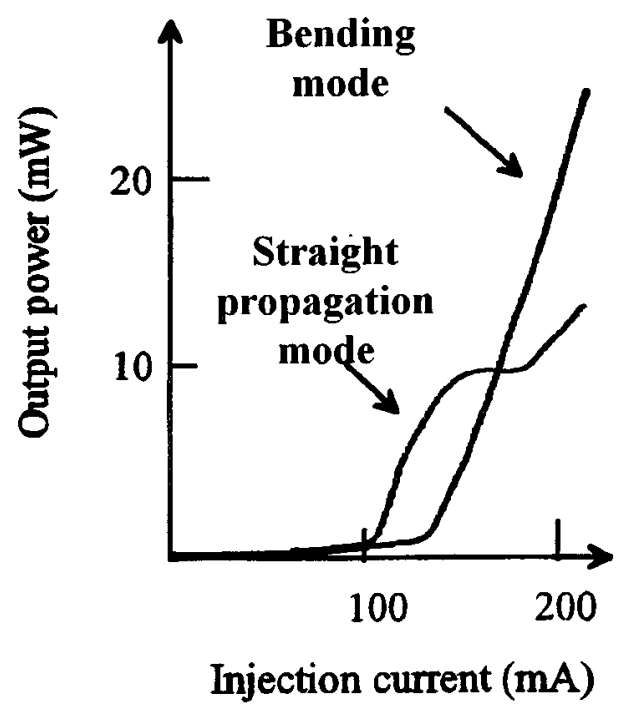

FIG. 5. $L-I$ curves of the optical switching device with the bending mode tuned to the wavelength $822.5 \mathrm{~nm}$.

In conclusion, a new type of optical switching device with a shallow-etched bending ridge waveguide is developed. With the weakly index-guided waveguide, the laser beam could possibly propagate along the bending direction or the straight direction. Switching between the two directions is observed. In addition, the switching is found to depend on the spectral separation of the two modes because the devices are fabricated on the substrate with dual quantum wells of different widths. As the carriers reside in the same well, their strong interaction causes a strong gain competition and so leads to an obvious switching behavior. On the other hand, if the carriers reside in different wells, their interaction is weak. As a consequence, the gain competition between the two modes is weak and no obvious switching behavior is observed.

The authors would like to acknowledge help from Professor Hao-Hsiung Lin at the Department of Electrical Engineering, National Taiwan University, and Dr. Wei Lin at the Photonic Technology Research Laboratory, Telecommunication Laboratories, Chung-Li, Taiwan, R.O.C. This work is supported in part by National Science Council, Taipei, Taiwan, R.O.C., under Contract No. NSC86-2112-M-002-025.

${ }^{1}$ K.-Y. Liou, C. A. Burrus, U. Koren, and T. L. Koch, Appl. Phys. Lett. 51, 1777 (1987).

${ }^{2}$ H. Shoji, Y. Arakawa, and Y. Fujii, IEEE Photonics Technol. Lett. 2, 109 (1990).

${ }^{3}$ Koji Nonaka, Yoshio Noguchi, Hiroyuki Tsuda, and Takashi Kurokawa, IEEE Photonics Technol. Lett. 7, 29 (1995).

${ }^{4}$ Y. C. Chen and J. M. Liu, Opt. Quantum Electron. 19, S93 (1987).

${ }^{5}$ H. Tanaka, J. Shimada, and Y. Suzuki, Appl. Phys. Lett. 64, 158 (1994).

${ }^{6}$ H. Uenohara, Y. Kawamura, H. Iwamura, K. Nonaka, H. Tsuda, and T. Kurokawa, Electron. Lett. 28, 1973 (1992).

${ }^{7}$ John E. Johnson and C. L. Tang, Appl. Phys. Lett. 63, 3273 (1993).

${ }^{8}$ B. B. Jian, Electron. Lett. 32, 1923 (1996).

${ }^{9}$ Ching-Fuh Lin, Master dissertation, Cornell University, Ithaca, New York, 1989.

${ }^{10}$ Ching-Fuh Lin and Chaur-Shiuann Juang, IEEE Photonics Technol. Lett. 8, 206 (1996).

${ }^{11}$ A. E. Siegman, Lasers (University Science Books, 1986).

${ }^{12}$ Ching-Fuh Lin, Bor-Lin Lee, and Po-Chien Lin, IEEE Photonics Technol. Lett. 8, 1456 (1996). 